\title{
SOME EXTENSION THEOREMS FOR REGULAR MAPS OF STEIN MANIFOLDS
}

\author{
BY CHESTER SEABURY
}

Communicated April 22, 1974

The central result of this paper is an analogue, in the category of complex manifolds and holomorphic maps, of the tubular neighborhood theorem. The following theorems are proved.

Theorem A. Let $S$ be a Stein manifold and let $f: S \rightarrow M$ be a holomorphic embedding. Let $K \subset S$ be compact and let $N_{f}$ be the normal bundle of $f$. We identify $S$ with the zero section of $N_{f}$ Then there is a neighborhood $U$ of $K$ in $N_{f}$ and a holomorphic embedding $F: U \rightarrow M$ such that $F \mid U \cap S=f$.

THEOREM B. The word "embedding" can be replaced by the word "immersion" in the above theorem.

THEOREM C. Let $f: S \rightarrow M$ be a holomorphic map, where $S$ is Stein, such that $f$ is regular at $x_{0} \in S$. Let $K \subset S$ be compact with $x_{0} \in$ $K$. Then there is a trivial bundle $\widetilde{A}$ over $S$, $\operatorname{dim}_{\mathrm{C}} \widetilde{A}=\operatorname{dim}_{\mathrm{C}} M$, a neighborhood $U$ of $K$ in $\widetilde{A}$, and a holomorphic map $F: U \rightarrow M$ such that $F \mid U \cap S=f$ and $F$ is regular at $x_{0}$ (again $S$ is identified with the zero section of $\widetilde{A}$ ).

When $M$ above is itself a Stein manifold, Theorems A and B are known and were proved by Forster and Ramspott. So the main effort of our work is to construct a strictly plurisubharmonic function $\phi$ on a neighborhood of $f(K)$ (in Theorem A) such that $\phi^{-1}((-\infty, b])$ is compact for all $b \in R$. Theorem $\mathrm{B}$ follows then from Theorem $\mathrm{A}$ by a well-known result. Theorem $\mathrm{C}$ follows from Theorem A by embedding a neighborhood of $g(K)$ in $S \times M$ in some $C^{q}(g(x)=(x, f(x)))$ and using the holomorphic retraction theorem and other standard results.

A special case of Theorem A, namely the case where $S$ is a disc, can

AMS (MOS) subject classifications (1970). Primary 32H99, 32C10. 
be applied to show that the differential metric associated to the Kobayashi metric on a complex manifold is upper semicontinuous.

\section{BIBLIOGRAPHY}

1. O. Forster and K. J. Ramspott, Analytische Modulgarben und Endromisbündel, Invent. Math. 2 (1966), 145-170. MR 36 \#1702.

2. R. G. Gunning and $\mathrm{H}$. Rossi, A nalytic functions of several complex variables, Prentice-Hall, Englewood Cliffs, N. J., 1965.

3. L. Hörmander, An introduction to complex analysis in several variables, Van Nostrand, Princeton, N. J., 1966. MR 34 \#2933.

4. H. L. Royden, Remarks on the Kobayashi metric, Proc. Maryland Conf. on Several complex variables, Lecture Notes in Math., vol. 185, Springer-Verlag, New York, 1971.

5. - The extension of regular holomorphic maps, Proc. Amer. Math. Soc. 43 (1974), 306-310.

DEPARTMENT OF MATHEMATICS, UNIVERSITY OF CALIFORNIA, IRVINE, CALIFORNIA 92664 